\title{
Are Epidemiological Approaches Suitable to Study Risk/Preventive Factors for Human Birth Defects?
}

\author{
Sonia Hernandez-Diaz • Anna Sara Oberg
}

Published online: 27 January 2015

(C) Springer International Publishing AG 2015

\begin{abstract}
Birth defects are a major cause of infant morbidity and mortality and contribute substantially to long-term disability. One out of every 33 babies is born with some type of birth defect. Despite decades of research on environmental, behavioral, and genetic risk factors, the vast majority of birth defects still occur without a known cause. It is possible that birth defects are largely stochastic (and unavoidable) events, at which efforts to investigate their causes would be futile and unjustified. In this commentary, we argue for the continued research into risk/ preventive factors of human birth defects, and outline why epidemiological studies are suitable for such endeavors. First, we discuss what factors to target (genetic or environmental) and how to define the pertinent research questions. Then, we present a short review of both epidemiological contributions in the past and approaches to advance the field in the future. After considering also their limitations, we conclude that modern epidemiologic approaches are invaluable to advance our understanding of risk factors for human birth defects, and that interdisciplinary collaborations will also be essential to further our knowledge.
\end{abstract}

Keywords Congenital malformations $\cdot$ Birth defects $\cdot$ Risk factors $\cdot$ Epidemiology $\cdot$ Observational studies

This article is part of the Topical Collection on Reproductive and Perinatal Epidemiology

S. Hernandez-Diaz $(\bowtie) \cdot$ A. S. Oberg

Department of Epidemiology, Harvard School of Public Health, 677

Huntington Avenue, Boston, MA 02115, USA

e-mail: shernan@hsph.harvard.edu

A. S. Oberg

e-mail: oberg@hsph.harvard.edu

\section{Introduction to the Scope of the Commentary}

This era of austerity has forced funding agencies to constantly consider where to spend the scarce resources, and the resulting harsh trade-offs require prioritizing certain fields of study over others. Investigators have to anticipate and follow the funders' directions; or take the risk and continue doing what they think is important, or simply know how to do. In that sense, there is competition both between and within fields of research. Shall all investigators go after popular topics such as obesity, or are orphan disorders such as many specific birth defects also relevant? Within birth defects research, shall we focus on genome analyses or on non-genetic causes; on animal models or on human studies? Or, perhaps, to be more appropriate, shall we discuss what the most pressing questions are first, and which approaches can provide valid answers to these questions second? Inspired by a workshop on "Developing an Interdisciplinary Research Agenda for Genetics of Birth Defects" held at the US National Institutes of Health in 2014, we share some of our thoughts below.

First, Is There Anything to Study or are Most Birth Defects Stochastic Events?

To discuss whether epidemiologic methods are appropriate to study birth defects, we first have to decide whether we want to spend the limited research funding available to investigate birth defects at all. Let's briefly review the burden and origins of birth defects: One out of every 33 babies is born with some type of birth defect [1]. Deaths due to birth defects account for more than $21 \%$ of all infant deaths, making them the leading cause of infant mortality. Birth defects are also associated with serious and often life-long disabilities; they are a leading cause of years of potential life lost, and account for much of the disability in children in the USA and most developed countries. Worldwide, congenital anomalies are the tenth leading 
cause of loss of disability-adjusted life years, accounting for $2.9 \%$ of all years of life lived with disabilities $[2,3]$. To achieve any reduction of this substantial infant mortality and childhood disability, we need to understand why and how birth defects occur. But, can we?

Some identified causes of congenital malformations include chromosomal defects, single gene defects, and a few environmental agents. However, despite decades of research on environmental, behavioral, and genetic risk factors, the vast majority (estimated at two thirds) of birth defects still occur without known cause [4]. Embryological processes are incredibly complex and inevitably subject to imperfections. In fact, as fundamental as biological variability is, so are the intrinsic errors of the processes. If birth defects are largely the result of stochastic, unavoidable events, then efforts to investigate their causes will be futile and unjustified. However, the existence of known clearly teratogenic agents suggests that others may remain unidentified. Moreover, deviant patterns of occurrence - across time, or between/within populations-also points toward non-stochastic influences. If research to date has allowed us to explain a third of human birth defects, advances in science (technology, methods, data) should be able to explain more of such probabilistic events, particularly when the causes are either commonly occurring, or rare but with high penetrance. Unless we believe that the majority of defects are a result of nature playing dice, identifying and understanding risk/preventive factors should have great implications for prevention and/or management of highrisk pregnancies. Conclusion: With only a small fraction of defects possible to predict or prevent with current knowledge, and all of the remainder unlikely to be just stochastic, it seems imperative to promote further research to identify the risk/ preventive factors for human birth defects.

\section{Shall we Focus on Genetic or Non-Genetic Causes?}

It is widely accepted that deterministic causes of birth defects are genetic, environmental or, most likely, a combination of the two (i.e., multifactorial) [5]. However, despite this prevailing idea, there is insufficient evidence for us to estimate the specific contribution of genes, environment, or their interaction. Although it is widely accepted that some structural defects recur in families [6-13], very few large population-based studies have attempted to use information on familial clustering to quantify the genetic versus environmental influence for the liability of malformations. To inform future directions for both funders and researchers, efforts to quantify the relative contributions of genetic and/or environmental factors to birth defect etiology should be of top priority.

Since little can be done (for now) to modify genetic factors, prevention depends upon the detection of environmentally determined, modifiable teratogens. However, the genetic background may also be relevant for prevention since it may interact with other factors, which can themselves be modified. For example, among the few teratogens that have been identified are traditional anticonvulsants [14, 15], yet far from all fetuses exposed to anticonvulsants will develop malformations. A case report of anticonvulsant-exposed dizygotic twins has showed that they can be discordant in physical features [16], and recent observations in pregnancy registers in the UK [17] and Australia [18] also suggest that the risk of anticonvulsant embryopathy may depend on genetic susceptibility to the teratogenic effect of these drugs. Conclusion: Without a formal quantification of the relative contribution of genetic and environmental causes, or their interaction, any prioritization will be a subjective preference of the decision maker. Epidemiological studies allow identification of both genetic and environmental risks on the population level; and some may allow the evaluation of gene-environment interactions as well. Population-based family designs can also be used to answer questions about genetic vs. environmental contributions to the (population) variation in birth defects, providing vital information for future research directions.

What are the Pertinent Questions from a Biologic, Clinical, or Public Health Perspective?

If we agree it is important to investigate birth defects, the next step will be to identify what the pertinent questions are. From a public health perspective, the most important aim should be to (1) identify the modifiable causes of birth defects, which will allow intervention and prevention of cases (e.g., by avoiding environmental exposures - teratogens). Additionally, it should be of great clinical value to (2) identify predictors of birth defects-including potential genetic influencewhich can help inform family planning (e.g., from quantification of absolute risk in specific human populations) and reduce morbidity through targeted screening and early intervention. Conclusion: The most pressing question may be how to prevent, or at least predict, birth defects.

\section{How can we Answer These Questions?}

Given that we agree on the pertinent questions, what would be the most suitable approaches to study them? Toxicological or in vitro studies provide limited information since we rarely know the specific teratogenic mechanisms [19]. A classic example is thalidomide, for which these mechanisms have remained unknown for decades. Animal models have provided vital insights to our understanding of common aspects of embryogenesis, and allow manipulations that would never be feasible in human studies. However, sometimes results are not directly translated into human risk because of considerable variations in teratogenic effects between mammalian species $[20 \bullet, 21]$. In humans, clinical trials are often unfeasible because most risk factors are non-randomizable for logistic (e.g., 
genes) or ethical (e.g., smoking) reasons. Information from randomized experiments in humans would only be available either as a secondary safety outcome or in the few instances where they test preventive agents (e.g., folic acid supplementation) [22]. However, the former usually exclude women who might become pregnant, particularly if there is any suspicion of adverse effects from animal studies. Therefore, we are mostly left with observational studies, briefly summarized in the following sections.

\section{What Epidemiological Designs are Available and/or Best?}

General support for epidemiological approaches can be obtained from reviewing the historical evidence. For major teratogens such as thalidomide, which increases the risk of specific malformations more than 100 -fold, clinical observations were able to identify clusters of exposed and affected motheroffspring pairs [23-25]. Even then, over 10,000 children had been affected by the time the drug was retracted from the market. Unlike thalidomide, most known teratogens will have a more modest risk, making their identification more challenging [26•]. To avoid another thalidomide tragedy, we now have proactive surveillance systems for pharmaceuticals, described below, which have helped to successfully identify also more modest teratogens (e.g., traditional anticonvulsants) [27]. Some epidemiological studies have been specifically designed to study birth defects, such as the large cohort study of over 50,000 pregnancies in the US Collaborative Perinatal Project [28]. Despite the large sample size, this study was nevertheless unable to evaluate most specific drugs, given the relatively small number of women exposed and the relatively rare outcomes under consideration [29]. Nowadays, cohort studies commonly focus on pregnancies specifically exposed to the factor (drug) of interest, i.e., pregnancy registries [30]. Yet, it still takes time to find and enroll enough numbers of exposed women soon after conception; and the registries often have limited sample sizes to look at specific malformations. For specific malformations, we favor case-control studies of birth defects, which would be more efficient if the exposure is not too infrequent [31]. Observations from these epidemiological designs have contributed to establish what is arguably the flagship for birth defects prevention, namely periconceptional folic acid supplementation to prevent neural tube defects (NTDs) [32, 33]. One lesson learned from these folic acid studies is that well-conducted observational studies can be as valid as randomized clinical trials to identify preventive factors for birth defects [34]. Finally, in the last decade, epidemiologists are increasingly using information from health care databases, i.e., administrative claims files, electronic medical records, or well-established population registers of health and demographic characteristics (e.g., from Scandinavian countries) $[35,36]$. These sources were not specifically designed to study birth defects, but efforts to utilize such prospectively collected health care data can still be desirable with respect to costs, numbers of pregnancies included, validity (e.g., no recall bias) and generalizability (i.e., population based). Nowadays, with the new surveillance systems established, epidemiological studies may not only be as valid but also much more efficient than clinical trials to identify risk or preventive factors for human birth defects.

What Epidemiological Methods are Available and/or Best?

Strong risk factors can be identified with crude simple epidemiologic methods [26 ${ }^{\bullet}$. To rule out strong teratogenic effects (e.g., over $20 \%$ risk of malformations after prenatal exposure to thalidomide), enrollment of 100 exposed pregnancies in a simple uncontrolled cohort might suffice. This is because the effect of major teratogens is so large that it overwhelms the potential impact of common methodological biases on relative risks. However, weaker factors are more difficult to identify, and will require larger samples and accurate estimates, i.e., carefully designed studies. Validity is crucial for modest effects.

For these modest risks, it is important to stick to the following "good practice" principles: (I) Consider specific birth defects, (II) consider specific factors (e.g., individual drugs), (III) focus on the etiologically relevant gestational period, (IV) obtain accurate measures of exposure and outcome, (V) enroll enough subjects to attain sufficient statistical power, (VI) avoid preferential publication in the context of multiple comparisons, (VII) replicate studies to confirm or refute initial findings, (VIII) write a detailed protocol (well-designed study, well-specified comparisons, appropriate analytic plan, etc.) that could be registered or shared, (IX) careful publication (expose and discuss limitations, present sensitivity analyses that quantify uncertainty, rational interpretation of scientific evidence, etc), and (X) transparent communication to stakeholders.

In addition to these principles, we may sometimes need to implement modern epidemiologic approaches. For instance, to avoid selection bias (e.g., by not adjusting for intermediate variables), evaluate direct and indirect effects (e.g., mediation analyses), or reduce confounding (e.g., propensity scores or inverse probability weighting). As an example, modern causal approaches could help us understand the role of plurality and prematurity on the effect of assisted reproduction technology (ART) on certain birth defects (Fig. 1).

The direct acyclic graph (DAG)'s validity depends on the identification of the correct relationship between the factors (nodes) and inclusion of all potential common causes of the exposure and outcome of interest. Vital knowledge to inform the DAGs will often originate from a broad range of disciplines, from genetics to anthropology. Epidemiological studies can benefit from other approaches indirectly (i.e., using their results to inform hypotheses and designs) and directly 


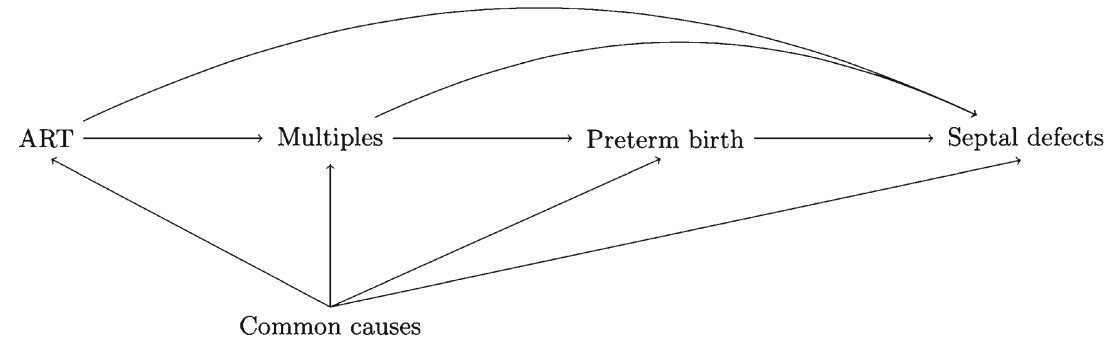

Fig. 1 Example of a direct acyclic graph (DAG) to apply causal thinking to birth defects. These causal networks can help us identify confounders (common causes) and mediators (multiples and preterm birth) of the exposure association to (specific) birth defects

(e.g., incorporating genetic and epigenetic testing). Therefore, interdisciplinary collaboration and integration of different approaches is essential for the understanding of causes and predictors of human birth defects.

In conclusion, all epidemiological studies should keep in mind some basic principles; some may need to consider advanced methods. We believe that epidemiological research is necessary to understand the origins of birth defects, to predict them, and to prevent them. However, it is not sufficient. Interdisciplinary collaborations will help move the field forward on more solid foundations.

\section{Counter Argument: Limitations of Epidemiological Studies}

While the prevention of NTDs is a success story, it may not necessarily be representative for other types of birth defects. Before the role of folic acid in NTD prevention was discovered, great variation in the occurrence of NTDs both over time and geographic areas had been described. Temporal trends and regional clusters would not be observed for completely stochastic events, thus pointing to the presence of one or more determinants that explained the variability. Further indications of an environmental influence came from observations that migrants acquired the risk in their new country of residence. In contrast to NTDs, most other birth defects show less variation in their occurrence across time and space. To infer from this observation alone that their occurrence is mostly stochastic may however be an oversimplification.

A major challenge to study birth defects in pregnancy cohorts is the right truncation of the cohort by the spontaneous abortion and therapeutic termination of pregnancies after prenatal diagnosis of a congenital malformation. The total risk of miscarriage is 12 to $15 \%$ in women at their healthiest years of reproduction (age 25 to 35), and it is reasonable to expect that severe and lethal malformations are common reasons for spontaneous abortions. In Europe, about $4 \%$ of pregnancies are terminated after prenatal diagnosis of birth defects and $18 \%$ of all fetuses with malformations are terminated following prenatal screening [37]. The proportion of terminations varies among specific defects [38•] and among populations. Thus, the commonly quoted prevalence of malformations at birth of around $3 \%$ does not reflect the incidence of malformations during embryo development. Inability to measure the true incidence can have important consequences for etiological studies of birth defects, particularly if the exposure influences the degree of selection (a problem not even randomized controlled trials can solve).

From a methodological standpoint, an optimal study design would allow consideration of all fetuses, terminated or not. For a majority of miscarriages, however, pathological analysis will never be feasible, as they may not even lead to a medical procedure. Those that occur under medical supervision are still unlikely to undergo diagnostic scrutiny with respect to specific type of defect(s). If studies generally need to be specific to a given defect to provide useful etiological information, inclusion of miscarriages would only add noise due to loss of specificity. If we believe that most teratogens influence specific processes, they may also mostly produce specific birth defects. Again, speculative, this could imply that stochastic events, including chromosomal aberrations, will in comparison be responsible for a greater portion of widestriking (multi-organ) disruptions, which are not compatible with life. If so, spontaneous abortions would represent a larger part of the stochastic quota and would, if included, rather lead to more noise in the quest to find causal factors. Also, from a public health perspective, miscarriages - sometimes not even noticed by the woman - and major birth defects may be completely different outcomes. While fertility and fetal viability are inarguably important, we may, as a society, be most interested in birth defects among those who survive the first trimester.

\section{Conclusions}

In summary, to quantify absolute risk in humans and inform couples based on their genetic and non-genetic characteristics we need prediction models based on data from a large number of humans (i.e., epidemiological studies). To identify causes of birth defects that may lead to prevention of cases in humans, we need causal inference thinking informed by data from a large number of humans (i.e., epidemiological studies). Epidemiologic approaches can be valuable for the 
identification of both teratogens and their interaction with genetic determinants. Thereby, they can further our understanding not only on currently unknown risk factors but also on known ones. To identify potential new teratogens, we have learnt from the thalidomide conundrum that we need to be proactive. To further our understanding of known risk factors, epidemiologic studies have been crucial to understand the relevance of folic acid pathways for birth defects. We learnt from folic acid that epidemiological plausibility can support biological findings as much as biological plausibility can support epidemiological ones. Although our aim has been to discuss the role of epidemiological studies, we also note that models from basic sciences and animal studies must be used to guide the design of epidemiologic studies. Hence, we conclude that yes, modern epidemiologic approaches are invaluable to furthering our understanding of risk factors for human birth defects. However, translational approaches and interdisciplinary collaborations will also be essential to further our knowledge.

Acknowledgments This work was inspired by the discussion at a workshop on "Developing an Interdisciplinary Research Agenda for Genetics of Birth Defects" held at the US National Institutes of Health in 2014. SHD gratefully acknowledges support from the US National Institute of Mental Health (Grant R01MH100216). The authors wish to acknowledge Dr. Allen Wilcox, who provided vital intellectual content both as discussant during the workshop and through revision of an early draft of the paper.

Disclosures The views expressed in this commentary are the views of the authors and do not necessarily reflect the views of the US National Institutes of Health.

\section{Compliance with Ethics Guidelines}

Conflicts of interest AS. Oberg and S Hernandez-Diaz declare no conflict of interest.

Human and Animal Rights and Informed Consent This article does not contain any studies with human or animal subjects performed by any of the authors.

\section{References}

Papers of particular interest, published recently, have been highlighted as:

- Of importance

1. Honein MA, Paulozzi LJ, Cragan JD, Correa A. Evaluation of selected characteristics of pregnancy drug registries. Teratology. 1999;60:356-64.

2. Centers for Disease Control. Economic cost of birth defects and cerebral palsy-United States, 1992. Morb Mortal Wkly Rep. 1995;44(37):694-9.

3. Murray CJ, Lopez AD. Global health statistics. Harvard: World Health Organization; 1996.
4. Nelson K, Holmes LB. Malformations due to presumed spontaneous mutations in newborn infants. N Engl J Med. 1989;320(1):19-23.

5. Elwood M, Little J, Elwood H. Epidemiology and control of neural tube defects, vol. 20. New York: Oxford University Press; 1992.

6. Lie RT, Wilcox AJ, Skjaerven R. A population-based study of the risk of recurrence of birth defects. N Engl J Med. 1994;331(1):1-4.

7. Skjaerven R, Wilcox AJ, Lie RT. A population-based study of survival and childbearing among female subjects with birth defects and the risk of recurrence in their children. N Engl J Med. 1999;340(14):1057-62.

8. Christensen K, Mitchell LE. Familial recurrence-pattern analysis of nonsyndromic isolated cleft palate - a Danish Registry study. Am J Hum Genet. 1996;58(1):182-90.

9. Christensen K, Schmidt MM, Vaeth M, Olsen J. Absence of an environmental effect on the recurrence of facial-cleft defects. $\mathrm{N}$ Engl J Med. 1995;333(3):161-4.

10. Burn J, Brennan P, Little J, et al. Recurrence risks in offspring of adults with major heart defects: results from first cohort of British collaborative study. Lancet. 1998;351(9099):311-6.

11. Oyen N, Boyd HA, Poulsen G, Wohlfahrt J, Melbye M. Familial recurrence of midline birth defects - a nationwide Danish cohort study. Am J Epidemiol. 2009;170(1):46-52.

12. Oyen N, Poulsen G, Boyd HA, Wohlfahrt J, Jensen PK, Melbye M. Recurrence of congenital heart defects in families. Circulation. 2009;120(4):295-301.

13. Pradat P. Recurrence risk for major congenital heart defects in Sweden: a registry study. Genet Epidemiol. 1994;11(2):131-40.

14. Holmes LB, Harvey EA, Coull BA, et al. The teratogenicity of anticonvulsant drugs. N Engl J Med. 2001;344:1132-8.

15. Hernandez-Diaz S, Smith CR, Shen A, et al. Comparative safety of antiepileptic drugs during pregnancy. Neurology. 2012;78(21): 1692-9.

16. Davidson DL. Letter to the editor. Discordant twins for neural tube defect on treatment with sodium valproate. Seizure. 2002;11(7): 445.

17. Campbell E, Devenney E, Morrow J, et al. Recurrence risk of congenital malformations in infants exposed to antiepileptic drugs in utero. Epilepsia. 2013;54(1):165-71.

18. Vajda FJ, O'Brien TJ, Lander CM, Graham J, Roten A, Eadie MJ Teratogenesis in repeated pregnancies in antiepileptic drug-treated women. Epilepsia. 2013;54(1):181-6.

19. Mitchell AA. Special considerations in studies of drug-induced birth defects. In: Strom BL, ed. Pharmacoepidemiology. Third ed: John Wiley \& Sons; 2000:750-763.

20. Holmes LB. Human teratogens: update 2010. Birth defects research. A Clin Mol Teratol. 2011;91(1):1-7. This manuscript reviews some of the known human teratogens, data sources where they can be identified and gaps of information. The author points out our inability to explain and predict teratogenic effects.

21. Wilson JG. Evaluation of human teratologic risk in animals. In: Lee DH, Hewson EW, Okun D, editors. Environment and birth defects. 1st ed. New York and London: Academic; 1973.

22. Laurence KM, James N, Miller MH, Tennant GB, Campbell H. Double-blind randomised controlled trial of folate treatment before conception to prevent recurrence of neural-tube defects. Br Med J. 1981;282(6275):1509-11.

23. McBride WG. Thalidomide and congenital abnormalities. Lancet. 1961;278(7216): 1358.

24. Burley DM, Lenz W. Thalidomide and congenital abnormalities. Lancet. 1962;279(7223):271-2.

25. Lenz W, Pfeiffer RA, Kosenow W, Hayman DJ. Thalidomide and congenical abnormalities. Lancet. 1962;279(7219):45-6.

26. Mitchell AA. Systematic identification of drugs that cause birth defects - a new opportunity. N Engl J Med. 2003;349(26):2556-9. The author proposes a surveillance system for teratogens and reviews the main study designs available to provide valid information, i.e., cohorts 
and case-control designs. The role, advantages, and limitations of each epidemiological approach are discussed.

27. Meador KJ, Pennell PB, Harden CL, et al. Pregnancy registries in epilepsy: a consensus statement on health outcomes. Neurology. 2008;71(14):1109-17.

28. U.S. Department of Health Education and Welfare PHS, National Institutes of Health. The collaborative perinatal study of the National Institute of Neurological Diseases and Stroke: the women and their pregnancies. 1972. DHEW Publication No. (NIH) 73-379.

29. Heinonen O, Slone D, Shapiro S. Birth defects and drugs in pregnancy. Littleton, Massachusetts: Publishing Sciences Group; 1977.

30. Lammer EJ, Chen DT, Hoar RM, et al. Retinoic acid embryopathy. N Engl J Med. 1985;313(14):837-41.

31. Werler MM, Louik C, Shapiro S, Mitchell AA. Prepregnant weight in relation to risk of neural tube defects. JAMA. 1996;275(14): 1089-92.

32. Mulinare J, Cordero JF, Erickson JD, Berry RJ. Periconceptional use of multivitamins and the occurrence of neural tube defects. JAMA. 1988;260(21):3141-5.

33. Milunsky A, Jick H, Jick SS, et al. Multivitamin/folic acid supplementation in early pregnancy reduces the prevalence of neural tube defects. JAMA. 1989;262(20):2847-52.
34. Prevention of neural tube defects: results of the Medical Research Council Vitamin Study. MRC Vitamin Study Research Group. Lancet. Jul 20 1991;338(8760):131-137.

35. Wettermark B, Zoega H, Furu K, et al. The Nordic prescription databases as a resource for pharmacoepidemiological research - a literature review. Pharmacoepidemiol Drug Saf. 2013;22(7):691-9.

36. Palmsten K, Huybrechts KF, Mogun H, et al. Harnessing the Medicaid Analytic eXtract (MAX) to evaluate medications in pregnancy: design considerations. PLoS ONE. 2013;8(6):e67405.

37. Dolk H, Loane M, Garne E. The prevalence of congenital anomalies in Europe. Adv Exp Med Biol. 2010;686:349-64.

38. Svensson E, Ehrenstein V, Norgaard M, et al. Estimating the proportion of all observed birth defects occurring in pregnancies terminated by a second-trimester abortion. Epidemiology. 2014;25(6):866-71. Using data from the Danish medical registries, the authors quantified the proportion of all fetuses with birth defects terminated in pregnancy. The proportion of birth defects terminated, i.e., unobserved at birth, varied by type of defect. The proportion was almost $50 \%$ for defects of the nervous system, but it was less than $10 \%$ for most types of birth defects. 